\title{
WINDS FROM RED GIANTS
}

Yu. A. Fadeyev

Astronomical Council of the USSR Academy

of Sciences, Pyatnitskaya 48 Moscow 109017 USSR

\section{Abstract}

Two principal mechanisms that may be responsible for mass loss from red giants are considered: shock wave-driven winds and radiatively (dust)-driven winds. Effect of the periodic shocks accompanying nonlinear oscillations of red giants is most prominent in the outer layers of the stellar atmosphere where shocks are able not only to expel gas but also increase gas density so that some molecular components become supersaturated. In O-rich stars the most abundant condensible species are silicon monoxide and iron, whereas in C-rich stars these are carbon, silicon carbide and iron.

\section{Introduction}

For nearly 30 years since Deutch (1956) interpreted the displacement of narrow absorption lines in the spectrum of $\alpha$ Her as a result of gas outflow, the red giants are known to lose their mass due to cool stellar winds. These winds are characterized by large mass fluxes and at the same time by relatively low velocities. According to Knapp and Morris (1985) the rates of mass loss from red giants are as high as a few $\times 10^{-4} \mathrm{M}_{\mathcal{O}} / y \mathrm{r}$, whereas the terminal wind velocities do not exceed $20 \mathrm{~km} / \mathrm{s}$ (Knapp 1985). At present four mechanisms are thought to drive stellar winds from cool low-surface gravity stars. These are (1) thermally driven winds; (2) Alfven wave driven winds; (3) shock-driven winds; and (4) radiatively driven winds. However in the present paper $I$ will discuss only two of these mechanisms: shock-driven and radiatively driven winds. This is due to the two following reasons. First, almost all red giants reveal the light variability because of stellar pulsations. The nonlinear pulsations of low-surface gravity stars are known to be accompanied by periodic shock waves that may expel gas from the outer stellar atmosphere. Second, almost all red giants are surrounded by circumstellar dust shells (Habing 1987). Therefore some role in mass loss may belong to momentum transfer from dust grains accelerated by stellar radiation to gas molecules. In order to estimate the contribution of such a wind to total mass loss one should consider in detail the dust formation process. In recent years some authors succeeded in application of the homogeneous nucleation theory to the problem of mass loss from cool stars. 
The presence of shock waves in atmospheres of pulsating stars is due to the existence of a critical frequency $\sigma$. for for sanding wave oscillations. For example, in the plane-parallel isothermal atmosphere oscillations exist in the form of standing wave if the dimensionless frequency

$$
\sigma=-\frac{2 \pi}{P}\left(\frac{\mathrm{R}_{\mathrm{ph}}}{3 G \mathrm{M}}\right)^{1 / 2}
$$

satisfies the following condition (Unno 1965):

$$
\sigma^{2}, \sigma_{c r}^{2}=\left(1+3 c^{2}\right) /(12 c)-4 / 3,
$$

where $P$ is the pulsation period, $G$ is the gravitation constant, $M$ is the mass of the star, $C=H_{p} / R p h$ is the ratio of the pressure scale height to the radius of photosphere. In other words, the critical frequency derives the upper frequency limit for perfect reflection of the pulsation wave at the stellar photosphere. Substituting the appropriate values of mass, radius and period in (1) and (2) one can readily find that standing wave transforms into progressive running waves for $C>0.01$. So, even in the framework of the linear theory the presence of running waves in the atmospheres of pulsating red giants seems to be inevitable since they are characterized by large values of $C$ ranged from a few percent to of about ten percent.

There are two most important consequences of the presence of periodic shock waves in the stellar atmospheres:

1) Periodic compression waves of finite amplitude enhance the atmospheric scale height (Whitney 1956). In pulsating low-surface gravity stars the dynamic scale height may by an order of magnitude exceed the static scale height (Fadeyev 1984; Willson and Bowen 1986). The distension of the stellar atmosphere by periodic shocks is very crucial for dust formation since in the hydrodynamically equilibrium stellar atmosphere condensation is impossible due to extremely low gas density. The tight interrelation between the shocks and the existence of circumstellar dust has been recently corroborated by a correlation between the infrared excesses and pulsation periods of red giants (De Gioia-Eastwood et al. 1981; Jura 1986).

2) Periodic shocks are able to expel gas from the outer stellar atmosphere. Let us assume that motion of the gas shell of initial radius $R_{0}$ and initial velocity $v_{0}$ is governed by gravitation only. Then the time needed for the shell to return to its origin is (Willson and Hill 1979):

$$
P_{\text {grt }}=2 R_{0} / v_{e}
$$

where

$$
\begin{aligned}
& b=\frac{\beta}{1-\beta^{2}}+\frac{1}{\left(1-\beta^{2}\right)^{3 / 2}} \tan ^{-1}\left(\frac{\beta^{2}}{1-\beta^{2}}\right)^{1 / 2} \\
& \beta=v_{0} / v_{e^{\prime}} v_{e} \text { is the escape velocity. The radius of the shell }
\end{aligned}
$$


averaged over the pulsation period increases with time if the ahell is not able to return to the level of its origin before the passage of the next shock, that is for $P_{g r t}>P$. For the sake of convenience, relation (3) can be rewritten in the terms of the pulation constant $Q$ and relative radius $x=R / R_{p h}$

$$
a\left(G M_{0} / 2 R_{0}^{3}\right)^{1 / 2}=x_{0}^{3 / 2}
$$

For the fixed value of the initial velocity $v_{0}$ which is interpreted as the postshock velocity, relation (5) determines the radius of the layer $R_{0}$ above of which the gravitation return time part exceeds the pulsation period. The advantage of the model by willson and fill (1979) is that far away from the star the mass loss is characterized by a steady flow rather than shock events.

However the existing estimates of the shock-driven mass loss rates are still uncertain due to their strong dependence on the cooling rates. According to Wood (1979) the mass loss rate may be ranged from $10^{-12} \mathrm{Mo} / \mathrm{yr}$ (isothermal shock) to $10^{-2} \mathrm{M}_{\odot} / \mathrm{Yr}$ (adiabatic shock). More certain estimates of mass loss rates might be derived from the models that take into account the interplay between the shock front propagating through the extended atmosphere, radiation field and atomic and molecular processes. Unfortunately no such a model has been constructed yet.

The most drastic effect on the losses of the thermal energy is due to dissociation of molecular hydrogen. According to Fox and wood (1985) as much as a half of the thermal energy behind the shock front is absorbed due to dissociation of $\mathrm{H}_{2}$ molecules. At the same time photodissociation of $\mathrm{H}_{2}$ molecules in the precursor causes retardation of the collisional ionization in the relaxation zone, whereas the precursor structure is very sensitive to the radiation flowing from the wake (Gillet and Lafon 1983; 1984). So, the self-consistent model of the radiative shock is urgently needed.

\section{Dust-driven winds}

Calculations of molecular equilibrium for gas with solar-like composition show that various molecular compounds condense in solid state when sas temperature drops below of 1000K-1500K (see e.g. Wood 1963; Lord 1965; Gilman 1969; Lattimer et al. 1978). However we should be interested only in a few condensible species that are characterized by both highest evaporation temperature and highest abundance. As was shown by Gail and Sedlmayr (1986) the most important condensible species are formed out a few most abundant elements that can be divided into the three following groupsi (1) hydrogen; (2) carbon, nitrogen, oxygen; (3) magnesium, silicon, sulphur, iron. Within each of these groups the elements are characterized by nearly comparable abundance. Other elements, such as natrium, aluminjum, calcium etc., can be neglected due to their lower abundances.

A sequence of condensates formed in the stellar atmosphere can be calculated provided that gas density and gas temperature $T$ are known as a function of the radius $R$. Let us assume that gas outflow is described by a spherically-symmetric steady-state continuity equation: 
and gas temperature is $T=T$ eff $W^{1 / 4}$, where $\dot{M}$ is the mass loss rate, $T_{\text {eff }}$ is the stellar effective temperature, $W$ is the radiation dilution factor. Then for a given gas velocity $V$ we can estimate the radius $R_{s a t}$ of a layer where the supersaturation ratio of a certain condensible species is $S=1$. This layer presents an inner boundary of the condensation region for a given molecular component since the condition of supersaturation $S>1$ fulfills at radij $\mathbf{R}>\mathbf{R}$

Table 1 and Table 2 give the radial distance $x$ expressed in units of $R_{p h}$ and gas temperature at the saturation level of the most abundant condensible species in $0-r i c h$ and C-rich stars with $M_{b o l}=-5 \mathrm{mag}$, respectively. It is assumed that gas velocity is equal to the local escape velocity, that is approximately is in the range from $10 \mathrm{~km} / \mathrm{s}$ to $20 \mathrm{~km} / \mathrm{s}$. Fortunately both the saturation radius $R_{\text {sat }}$ and gas temperature $T_{\text {sat }}$ only slightly depend on the expansion velocity. For example, the change in the ratio $\mathrm{v} / \mathrm{v}$ from 0.5 to 2.0 corresponds to the change in $R_{\text {sat }}$ and $T$ sat less than $5 \%$

Table 1. Properties of saturation levels at $[C] /[0]=0.5$

\begin{tabular}{|c|c|c|c|c|c|c|c|c|c|}
\hline \multirow{2}{*}{$T_{\text {eff }}$} & \multirow{2}{*}{$\log \dot{M}$} & \multicolumn{2}{|c|}{ MgO } & \multicolumn{2}{|c|}{$\mathrm{Fe}$} & \multicolumn{2}{|c|}{ MgS } & \multicolumn{2}{|c|}{ sio } \\
\hline & & $x$ & $\mathbf{T}$ & $x$ & $\mathrm{~T}$ & $x$ & $T$ & $x$ & $T$ \\
\hline \multirow[t]{3}{*}{3000} & -3 & 3.26 & 1182 & 4.04 & 1059 & 5.46 & 910 & 6.38 & 841 \\
\hline & -5 & 3.87 & 1083 & 5.01 & 950 & 6.49 & 834 & 7.78 & 761 \\
\hline & -7 & 4.53 & 1000 & 6.07 & 862 & 7.71 & 765 & 9.31 & 696 \\
\hline \multirow[t]{3}{*}{3500} & -3 & 4,41 & 1182 & 5.48 & 1060 & 7.42 & 910 & 8.66 & 841 \\
\hline & -5 & 5.24 & 1083 & 6.80 & 950 & 8.82 & 834 & 10.58 & 761 \\
\hline & -7 & 6.15 & 1000 & 8.25 & 862 & 10.48 & 765 & 12.66 & 696 \\
\hline
\end{tabular}

Table 2. Properties of saturation levels at $[C] /[0]=2.0$

\begin{tabular}{|c|c|c|c|c|c|c|c|}
\hline \multirow{2}{*}{$T_{\text {eff }}$} & \multirow{2}{*}{$\log \dot{\mathrm{M}}$} & \multicolumn{2}{|c|}{ C } & \multicolumn{2}{|c|}{ Sic } & \multicolumn{2}{|c|}{$\mathrm{Fe}$} \\
\hline & & $x$ & $\mathbf{T}$ & $x$ & $\mathrm{~T}$ & $x$ & $\mathrm{~T}$ \\
\hline \multirow[t]{3}{*}{3000} & -3 & 1.53 & 1771 & 2.15 & 1469 & 4.04 & 1059 \\
\hline & -5 & 1.73 & 1653 & 2.55 & 1343 & 5.01 & 950 \\
\hline & -7 & 1.99 & 1530 & 3.00 & 1235 & 6.07 & 862 \\
\hline \multirow[t]{3}{*}{3500} & -3 & 2.02 & 1772 & 2.88 & 1470 & 5.48 & 1060 \\
\hline & -5 & 2.30 & 1654 & 3.43 & 1343 & 6.80 & 950 \\
\hline & -7 & 2.66 & 2530 & 4.05 & 1235 & 8.25 & 863 \\
\hline
\end{tabular}

In O-rich stars almost all atoms of carbon are tied in molecules of carbon monoxide co so that the condensible species are mainly refractory oxides and 
atomic iron. The existence of circumstellar silicate grains is compelling oue to the si-o stretching spectral feature observed in either emission or absorption at $9.7 \mu \mathrm{m}$, and the weaker o-si-o bending feature observed always in emission at $18.0 \mathrm{Hm}$. The existence of MgS dust particles in o-rich stars is questionable because this material may be rapidly destroyed via reactions with $\mathrm{OH}$ and $\mathrm{H}_{2} \mathrm{O}$ molecules (Nuth et al. 1985).

In crich stars almost all atoms of oxysen are blocked in co molecules, whereas the large fraction of carbon is tied in molecules $\mathrm{C}_{2} \mathrm{H}$ and $\mathrm{C}_{2} \mathrm{H}_{2}$. The rest of carbon exists in the form of free atoms and molecules of silicon carbide sic. Both carbon and silicon carbide are the most abundant condensible species in C-rich stars. The presence of $\mathrm{SiC}$ circumstellar grains is compelling due to the $11.3 \mathrm{\mu m}$ spectral feature identified firstly by Treffers and Cohen (1974).

Dust formation is an irreversible, non-equilibrium process so that in order to derive the final radii and number densities of dust grains one should numerically integrate the differential equations describing the mass exchange between gas phase and solid state. It is such an approach that was used by Fix(1969), Woodrow and Auman(1982), Fadeyer (1983; 1987), Gail et al. (1984), Gail and Sedimayr (1985; 1986; 1987) for calculation of duat formation in circumstellar environments. All these studies are based on the classical theory of homogeneous nucleation (see e.g. Feder et al. 1966; Abraham 1974 for reviews) and the assumption of spherical dust grains. Results of these calculations can be summarized in a following way.

When the supersaturation ratio $\mathbf{s}$ becomes greater than unit, the small liquid droplets (i.e. molecular clusters) commence to appear. Almost all the droplets are immediately destroyed due to evaporation and only small fraction of the droplets (critical clusters) with radii greater than a critical radius $r_{\text {n }}$ have a chance to survive and grow by accretion of vapor molecules (monomers) onto their surface. It is assumed that macroscopic thermodynamics is applied to the critical clusters that are considered as liquid droplets containing the large number of monomers, that is $n_{p} \gg 1$. The number of the critical clusters formed per unit time per unit volume is the nucleation rate $J$ so that the number density of dust grains is $\mathrm{N}_{\mathrm{d}}=\int \mathrm{Jdt}$. Expressions for calculation of the nucleation rate and other quantities can be found in the review paper by Draine (1981).

At the beginning of phase transition the nucleation rate quickly increases with time until growth of dust grains perceptibly depletes vapor. After that both the supersaturation ratio and the nucleation rate drop and phase transition is due to growth of dust grains. The existence of the maximum of the nucleation rate is the result of the competition between decreasing gas temperature and increasing mean collision time of monomers $t_{\text {col }}$ The interplay between these two effects accompanying expansion of gas can be described by a dimensionless parameter $\Lambda=t_{s a t} / t_{c o l}$ where $t_{s a t}$ is supersaturation ratio e-folding time. Clearly, phase transition negligibly departs from thermal equilibrium if $\Lambda \gg 1$. In this case the problem of dust formation is simplified since it can be reduced to the solution of algebraic equations (Yamamoto and Hasegawa 1977; Draine and Salpeter 1977). Unfortunately the condition $A \gg 1$ usually does not fulfill in circumstellar flows so that these approaches should be used with caution.

According to Fadeyev and Henning (1987) the final radii of 310 grains formed in O-rich stars with $M_{\text {bol }}=-5 \mathrm{mag}$ and $2500<\mathrm{T}$ eff $<3000 \mathrm{~K}$ relate to mass flux as 
$108 r=\left\{\begin{array}{l}4.38 \\ 4.45\end{array}\right\}+\left\{\begin{array}{l}0.78 \\ 0.70\end{array}\right\} 108 \dot{M}$

where $r$ and $\dot{M}$ are expressed in angstroms and $M_{0}$. The fitting coefficients were estimated for the surface tension energy $0=500$ erg/cm and 750 erg/ $\mathrm{cm}^{2}$, respectively.

The studies of carbon dust formation in c-rich stars have been recently done by Gail et al. (1984) and Gail and Sedlmayr (1985; 1987). They found that the final radii of solid carbon particles are in the range from $130 \mathrm{~A}$ to $670 \mathrm{~A}$ for mass loss rate ranged from $10^{-6} \mathrm{M}_{0} / \mathrm{Yr}$ to $2 \times 10^{-4} \mathrm{M} \mathrm{M}_{\mathrm{Yr}} \mathrm{The}$ existence of large dust grains is due to the fact that grain growth proceeds not only by accretion of free carbon atoms and $C_{2}, c_{3}$ molecules via the following exchange reactions:

$$
\begin{aligned}
& C_{n}+C_{2} H_{2}=C_{n+2}+H_{2}, \\
& C_{n}+c_{2} H=C_{n+2}+H,
\end{aligned}
$$

where $n$ is the number of carbon atoms in the dust grain.

The strict solution for the problem of the resistance to the motion of a small sphere moving through gas has been obtained by Baines et al. (1965). They considered both specular and diffuse reflection of the molecules at the surface of the sphere mass of which is large in comparison with the mean mass of gas molecules and the radius to be small compared with the mean free path of gas molecules. All these assumptions are applicable for circumstellar outflows. Fadeyev and Henning (1987) used these solutions for calculation of momentum transfer from silicate dust grains to gas molecules in cool 0-rich red giants with $M_{b o l}=-5 \mathrm{mag}$ and found that at the radial distances less than $10 R_{p h}$ the drift velocity of dust grains does not exceed $0.1 \mathrm{~km} / \mathrm{s}$. Gail and sedimayr (1986) studied dust formation in gas outflows from 0-rich red eiants with $L=10^{4} L_{\odot}, T_{\text {eff }}=2500 \mathrm{~K}$ and $M=10^{-5}, M_{\odot} / \mathrm{Yr}$. They found that at radial distances less than $32 \mathrm{R}_{\mathrm{ph}}$ the sas expansion velocity does not exceed $5 \mathrm{~km} / \mathrm{s}$. This is due to the small final radil of dust grains that are ranged from $10 \mathrm{~A}$ to $20 \mathrm{~A}$. In C-rich stars the terminal velocities are ranged from $8 \mathrm{~km} / \mathrm{s}$ to 30 $\mathrm{km} / \mathrm{s}$ for the mass loss rates ranged from $10^{-6} \mathrm{M} / \mathrm{Yr}$ to $2 \times 10^{-4} \mathrm{M}$ /yr, respectively.

\section{References}

Abraham F.F, 1974, Homogeneous Hucleation. Academic Press, New York. Baines M.J., Williams I.P., Asebiomo A.S, 1965, Mon. Not.R.astr.Soc., $130,63$. De Gioia-Eastwood K., Hackwell J.A., Grasdalen G.L., Gehrz R.D. 1981,

Astrophys.J. (Letters), 245, L75.

Deutch A.J. 1956, Astrophys.J., 123, 210.

Draine B.T. 1981, in Physical Processes in Red Giants, ed. I. Iben and A.Renzini,

D. Reide1, Dordrecht, p. 317.

Draine B.T., Salpeter E.E. 1977, J.Chem.Phys., 67, 2230. 
Fadeyev Yu.A. 1983, Astrophys.Space Sci., 95, 357.

Fadeyev YU.A. 1984, Astrophys. Space Sci., 100, 329.

Fadeyev Yu.A. 1987, in Circumstellar Matter, ed. I.Appenzeller and C.Jordan, D.Reidel, Dordrecht, p. 515.

Fadeyev Yu.A., Henning T. 1987, in preparation.

Feder J., Russel K.C., Lothe J., Pound G.M. 1966, Adv. Phys., 15, 11.

Fix J.D. 1969, Mon.Not.R. astr.Soc., 146, 37.

Fox M.W., Wood P.R. 1985, Astrophys.J., 279, 455.

Gail H.-P., Keller R., Sedlmayr E, 1984, Astron.Astrophys., 133, 320.

Gail H. -P., Sedlmayr E. 1985, Astron.Astrophys., 148, 183.

Gail H. -P., Sedlmayr E. 1986, Astron. Astrophys., 166, 225.

Gail H. -P., Sedlmayr E. 1987, Astron. Astrophys., 171, 197.

Gillet D., Laf on J.-P.J. 1983, Astron. Astrophys., 128, 53.

Gillet D., Lafon J.-P.J. 1984, Astron.Astrophys., 139, 401.

Gilman R.C. 1969, Astrophys.J. (Letters), 155, L185.

Habing H.J. 1987, in Circumstellar Matter, ed. I.Appenzeller and C.Jordan, D.Reidel, Dordrecht, p. 197 .

Jura M. 1986, Irish Astron.J., 19, 322.

Knapp G.R. 1985, Astrophys.J., 293, 273.

Knapp G.R., Morris M. 1985, Astrophys.J., 292, 640.

Lattimer J.M., Schramm D.N., Grossman L. 1978, Astrophys.J., 219, 230 .

Lord H.C. 1965, Icarus, 4, 279.

Nuth J.A., Donn B. 1982, Astrophys.J. (Letters), 257, L103.

Nuth J.A., Donn B. 1982, J.Chem. Phys., 77, 2639.

Nuth J.A., Donn B. 1983, J.Chem. Phys., 98, 1610.

Nuth J.A., Moseley S.H., Silverberg R.F., Goebel J.H., Moore W.J. 1985, Astrophys.J. (Letters), 290, L41.

Treffers R., Cohen M. 1974, Astrophys.., 188, 545.

Unno W. 1965, Publ. Astron.Soc.Japan, 17, 205.

Whitney C. 1956, Ann.d'Astrophys., 19, 34.

Willson L.A., Bowen G.H. 1985, Irish Astron.J., 17, 249.

Willson L.A., Hill S.J. 1979, Astrophys.J., 228, 854.

Wood J.A. 1963, Icarus, 2 , 152.

Wood P.R. 1979, Astrophys.J., 227, 220.

Wooddrow J.E.J., Auman J.R. 1982, Astrophys.J., 256, 247.

Yamamoto T., Hasegawa H. 1977, Prog. Theor.Phys., 58, 816. 